\title{
Erosion of the prismatic layer by the organic matrix during the formation of the nacre-prism transition layer in the shell of Pinctada fucata (Bivalvia, Mollusca)
}

\author{
LIU XiaoJun ${ }^{1}$, LIU Chang ${ }^{1}$, SUN Juan ${ }^{1}$, ZHOU YuJuan ${ }^{1}$, ZHENG GuiLan ${ }^{1}$, ZHANG GuiYou ${ }^{1}$, \\ WANG HongZhong ${ }^{1}$, XIE LiPing ${ }^{1,2 *}$ \& ZHANG RongQing ${ }^{1,2^{*}}$ \\ ${ }^{1}$ Institute of Marine Biotechnology, School of Life Sciences, Tsinghua University, Beijing 100084, China; \\ ${ }^{2}$ Protein Science Laboratory of the Ministry of Education, Tsinghua University, Beijing 100084, China
}

Received September 19, 2010; accepted December 21, 2010

\begin{abstract}
Three variants of the sequence of formation of the nacre-prism transition layer were observed in Pinctada fucata (Bivalvia, Mollusca) shells. In each case, the layer was formed by the organic matrix secreted by the mantle, together with the interprismatic organic envelope. The continuity of the organic phase throughout the shell was maintained as the new nacreous layer was formed on the nacre-prism transition layer. Changes in the interprismatic organic envelopes on either side of the nacre-prism transition zone indicated that the organic matrix of the nacre-prism transition layer becomes embedded into the organic phase of the prismatic layer. It is concluded that penetration and erosion of the prisms by the organic matrix generates a strong bond between the prismatic and nacreous layers.
\end{abstract}

mollusk shell, organic framework, structure bonding, organic macromolecules

Citation: Liu X J, Liu C, Sun J, et al. Erosion of the prismatic layer by the organic matrix during the formation of the nacre-prism transition layer in the shell of Pinctada fucata (Bivalvia, Mollusca). Chinese Sci Bull, 2011, 56: 869-876, doi: 10.1007/s11434-011-4348-8

Bivalve shells are composite bioceramics formed via the precise assembly of $\mathrm{CaCO}_{3}$ crystals within an organic matrix. The organic matrix is composed of macromolecules, principally chitin and matrix proteins. These matrix proteins have been isolated from bivalve shells as a whole or individually purified, and their interactions with crystals have been studied extensively [1-5]. The organic matrix plays a crucial role in shell formation. Water-soluble proteins induce crystal nucleation [1,6], inhibit crystal growth [7], and control crystal morphology [4], while water-insoluble proteins are mainly involved in construction of the organic framework. Thus, the organic matrix controls the formation of various types of $\mathrm{CaCO}_{3}$ crystals, and their organization, resulting in the different layers of the shell.

The pearl oyster Pinctada fucata is a typical bivalve

*Corresponding authors (email: rqzhang@mail.tsinghua.edu.cn; lpxie@mail.tsinghua.edu.cn) whose shell is composed of two distinct mineralized layers, the outer calcitic prismatic layer and the inner aragonitic nacreous layer. The prismatic layer is assembled on proteins produced by epithelial cells of the mantle margin, whereas the nacreous layer is laid down on proteins produced by the central mantle epithelium [8]. The two calcified layers are separated by a thin organic lamella, referred to in this paper as the "nacre-prism transition layer". The two calcified layers and the transition layer grow simultaneously as the shell expands and a "transition zone" between the two types of shell is always visible where the nacre-prism transition layer intersects with the inner surface of the shell (Figure 1). The transition zone advances outward as the shell grows, in the course of which a sequential change occurs in the mantle epithelium, from laying down of the prismatic layer, to secretion of the transitional layer, to production of nacre.

The nacre-prism transition layer is important because it 


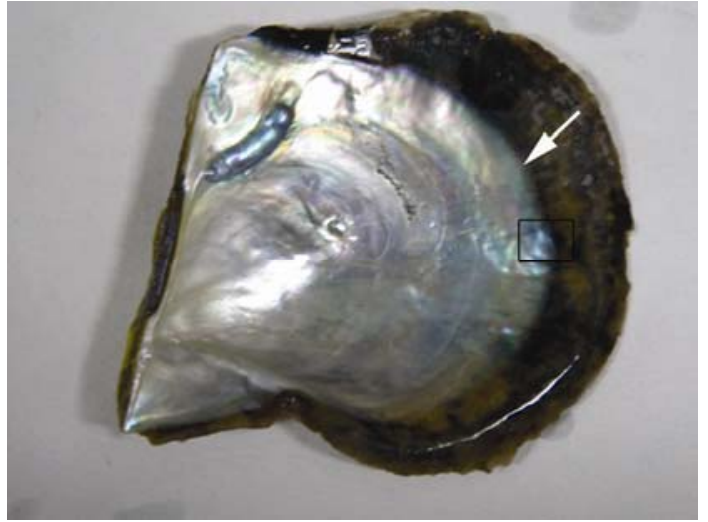

Figure 1 The inner surface of a Pinctada fucata shell. The nacre-prism transition zone is visible indicated (arrow and closed box).

provides the initial nucleating surface for aragonite and it alters the mode of shell growth. Great differences in mineralogy, microstructure, and organic matrix exist between the prisms and the nacre. For example, the major matrix proteins found specifically in the prismatic layer have a lower pI than those found only in the nacreous layer [9-13]. Thus, a series of changes must occur on the surface and within the prismatic layer as the nacre-prism transition occurs. However, little is known concerning these changes, and the mechanism by which these two different layers become closely joined remains uncharacterized.

We investigated this process by observing the changes that occur in the organic matrix during the formation of the nacre-prism transition layer in shells of P. fucata. Based on these observations, we propose an "embedment model" which explains the role of the organic matrix in the nacre-prism transition zone, and the strong adhesion between the prismatic and nacreous layers.

\section{Materials and methods}

Pearl oysters (Pinctada fucata) with wet weights of 30-40 g and shells $\sim 4.0-6.0 \mathrm{~cm}$ in diameter were collected from Guofa Pearl Farm in Beihai, Guangxi Province, China. Thirty-five $P$. fucata shells were cleaned with Milli-Q water and air dried. They were fractured into small fragments using liquid nitrogen and pieces containing the nacre-prism transition zone (Figure 1) were selected. Ten additional shells fractured as above but were observed without undergoing the EDTA treatment. To observe the changes that occur in the organic matrix in the prisms near the nacre-prism transition, 20 shell pieces were slightly decalcified in 5\% EDTA for $10 \mathrm{~min}$ and another 5 pieces were completely decalcified in 5\% EDTA for $3 \mathrm{~h}$. Ten shells were prepared similarly but were not subjected the EDTA treatment. After coating with gold, the inner surfaces of both treated and untreated shell pieces, and cross-sections of the untreated pieces, were examined using a scanning elec- tron microscope (SIRION 200, FEI Co.).

\section{Results}

\subsection{Three variants of the nacre-prism transition sequence}

The nacre-prism transition layer is an organic layer that covers the internal surface of the prismatic layer (Figure 2(a)). The transition layer provides a surface for polycrystalline aragonite growth [14]. We identified 3 different configurations of the nacre-prism transition zone, as described below.

(i) Increased thickness of the organic matrix forming the interprismatic organic envelopes. This form of the nacreprism transition layer has been reported in previous studies [14] and was observed here in slightly decalcified shells. The surfaces of the prisms adjacent to the nacre-prism transition zone were not completely visible and regions close to the interprismatic organic envelope were covered by organic matrix (Figure 2(b)). Because the mantle was secreting organic matrix onto the internal shell surface during the process, the surface of the interprismatic organic envelope was elevated (Figure 2(c)). Organic matrix was continuously added and, in surface view, appeared to converge toward the center of the prisms (Figure 2(b)) until the surface was entirely covered by the organic matrix. Thus, the nacre-prism transition layer was formed at the end of this process.

(ii) Transformation of the prismatic layer into the nacre-prism transition layer. This second type of transition layer formation was observed in untreated shells. In surface view, the shape of the prisms located just outside the nacreprism transition zone appeared to change (Figure 3(a) and (b)) and, extending further into the transition zone, the visible surface area of the prisms was reduced, or they disappeared completely; i.e. they were visible when still partially invested by the organic matrix but became invisible as they were covered by the matrix. The interprismatic organic envelope surrounding each prism seemed to be incorporated into the organic matrix (Figure 3(c)). Before this stage, the thickness of the interprismatic organic envelopes remained unchanged although their shape was distorted. Therefore, it appeared that new organic matrix was secreted onto the inner surface of the prismatic layer.

(iii) Decreased organic matrix in the interprismatic organic envelopes in the formation of the nacre-prism transition layer. This variation of transition layer formation was observed in slightly decalcified shells. On the inner surface close to the nacre-prism transition zone, the thickness of the organic envelope of prisms decreased (Figure 4(a)). The double-layered interprismatic organic envelopes were elevated above the surface in areas distant from the transition zone (Figure 4(b) and (c)), while in areas nearer to the transition zone, the surface was smooth (Figure 4(d)). This latter phenomenon reflected a decrease in the thickness of the 

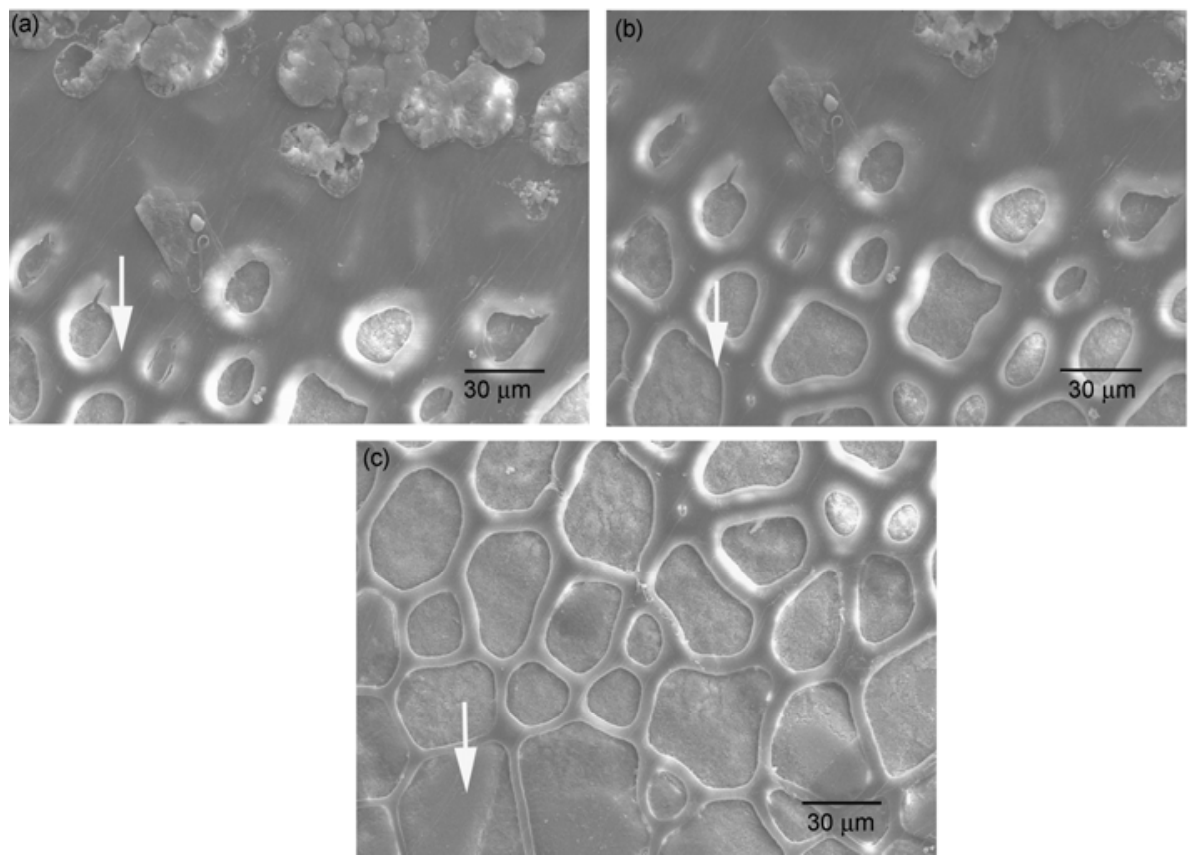

Figure 2 The first type of nacre-prism transition layer formation. (a) An organic layer representing the nacre-prism transition layer forms on the prismatic layer; (b) the diameter of the visible surface of the prisms decreases before the formation of the nacre-prism transition; (c) the addition of the organic matrix to the interprismatic organic envelope of the prismatic layer near to the transition zone. The shell was slightly decalcified in 5\% EDTA for 10 min. The white arrows indicate the growth direction of the shell.
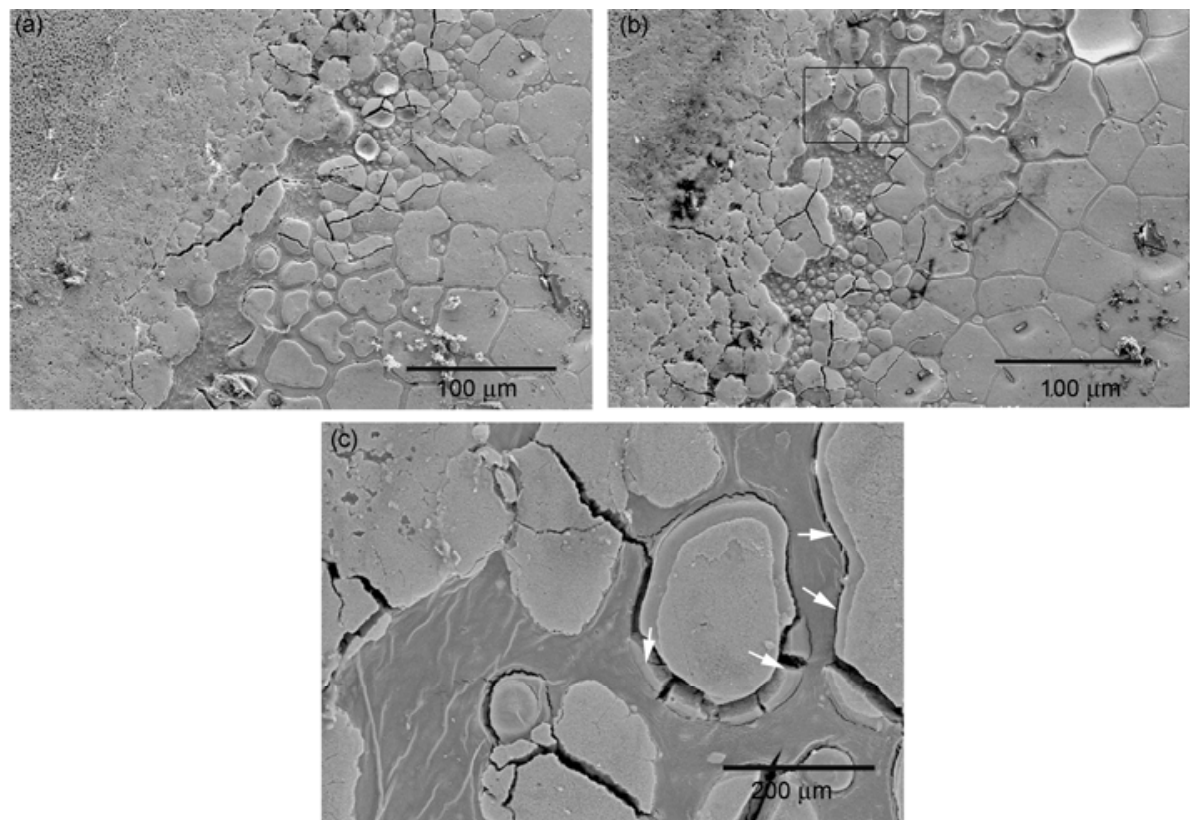

Figure 3 The second type of nacre-prism transition layer formation. (a) and (b) The transformed prisms before the formation of the nacre-prism transition. (c) Enlargement of the area indicated in (b); dissolution of the interprismatic organic envelope as the nacre-prism transition layer forms. The growth direction of the shell is from left to right.

organic matrix of the interprismatic organic envelopes and the retreat of surface of the interprismatic organic envelopes below the inner surface of the shell (Figure 4(d)). In areas closer to the nacre-prism transition zone, the prisms appeared to grow over both layers of organic matrix of the interprismatic envelopes, leaving only the central part ex- posed (Figure 4(e)). In these regions, the prisms were not different in shape but, adjacent to the transition zone, the visible surface area of the prisms decreased suddenly and the space between neighboring prisms was filled with the organic matrix (Figure 4(f)). This newly secreted organic matrix also lay below the inner surface of the shell. 

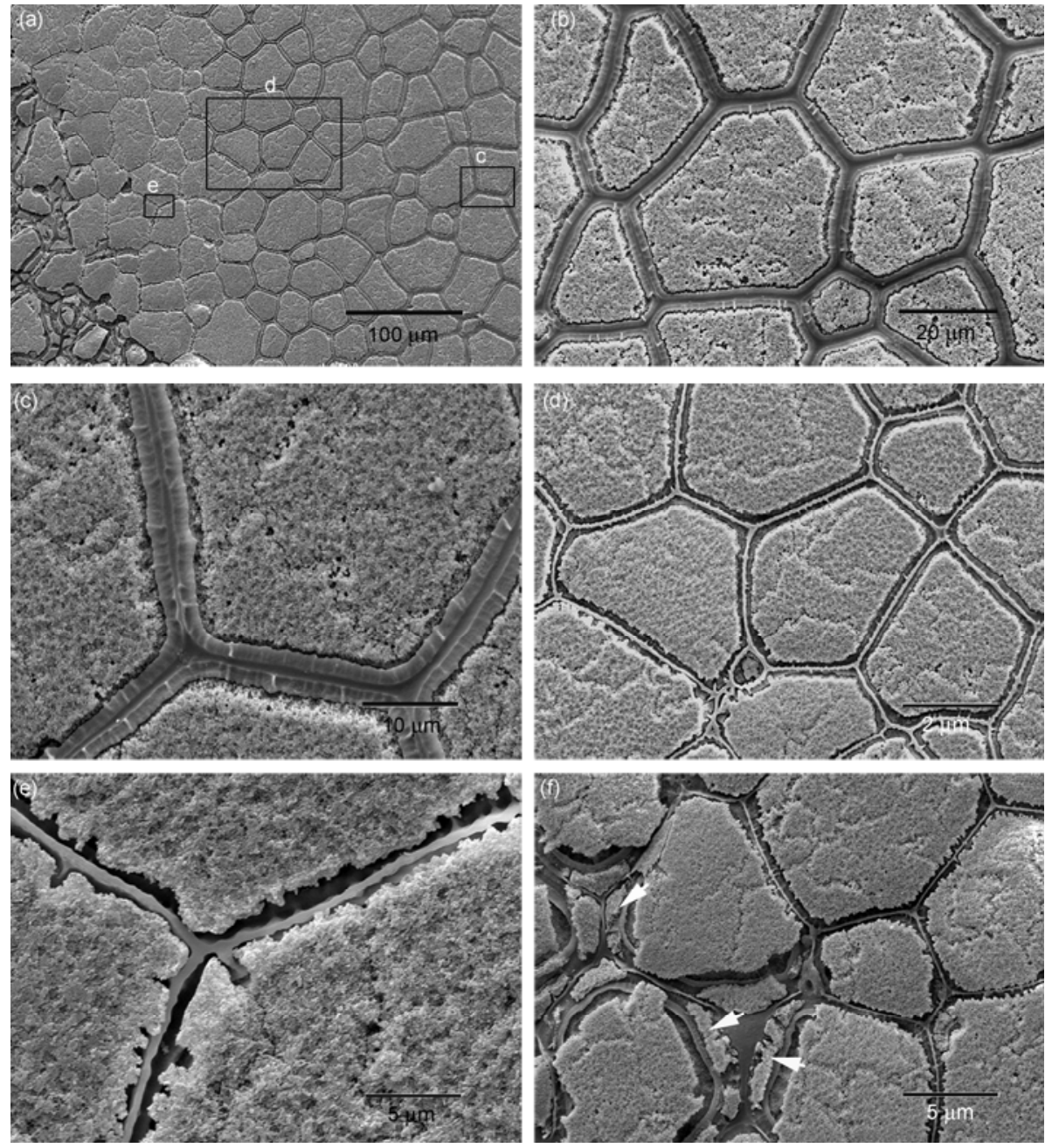

Figure 4 The third type of nacre-prism transition layer formation. (a) The prismatic layer in the transition zone. Areas enlarged in panels (c), (d) and (e) are indicated. The shell was slightly decalcified in 5\% EDTA for 10 min. (b) The prismatic layer distant from the figure (a). (c) The interprismatic organic envelopes of the prisms far from the transition. (d) The prismatic layer near the transition zone. (e) The interprismatic organic envelopes of the prisms near the transition zone. (f) The disappearance of the interprismatic organic envelopes on the surface of the organic matrix composing the transition layer. The growth direction of the shell is from right to left.

\subsection{Changes in the organic matrix in cross-sections of the shell}

Examination of cross-sections of an untreated shell revealed that a rapid change must take place within the organic matrix of the prismatic layer before nacre is formed (Figure $5(\mathrm{a})$ ). The surfaces of the prisms in the prismatic layer were flat and smooth in regions outside the transition zone (Figure $5(\mathrm{~b})$ ). Close to the nacre-prism transition zone, the prismatic surface began to be covered by organic macromolecules and became rough in texture (Figure 5(c)). In the transition zone itself a thicker layer of organic macromolecules extended over the surface (Figure 5(d)). This change occurred not only on the surface but penetrated into the interprismatic organic envelopes.

In the transition zone further toward the nacreous layer, it was observed that the organic matrix of the interprismatic organic envelopes was much increased and the boundaries between neighboring prisms were not visible (Figure 5(d)).
Further still into the transition zone, a complete organic layer was present (Figure 5(e)). This layer partly extended below the tips of the prisms, which were visible at the growth front in the nacre-prism transition zone (Figure 6, white lines and arrows). The height of the organic layer from the inner surface of the shell to the prisms was $\sim 20$ $\mu \mathrm{m}$. As the nacreous layer increased in thickness, the organic matrix in the transition layer continuously decreased (Figure 5(e) and (f)). In the fully formed nacreous layer, the organic matrix was even further reduced (Figure 5(f) and (g)).

\section{Discussion}

\subsection{The continuum of the organic phase in the whole shell}

Although the prismatic layer and the nacreous layer differ in mineral morphology, in organic matrix composition, and in crystal assembly, the two layers share the common characteristic that the crystals are embedded in an organic matrix. 


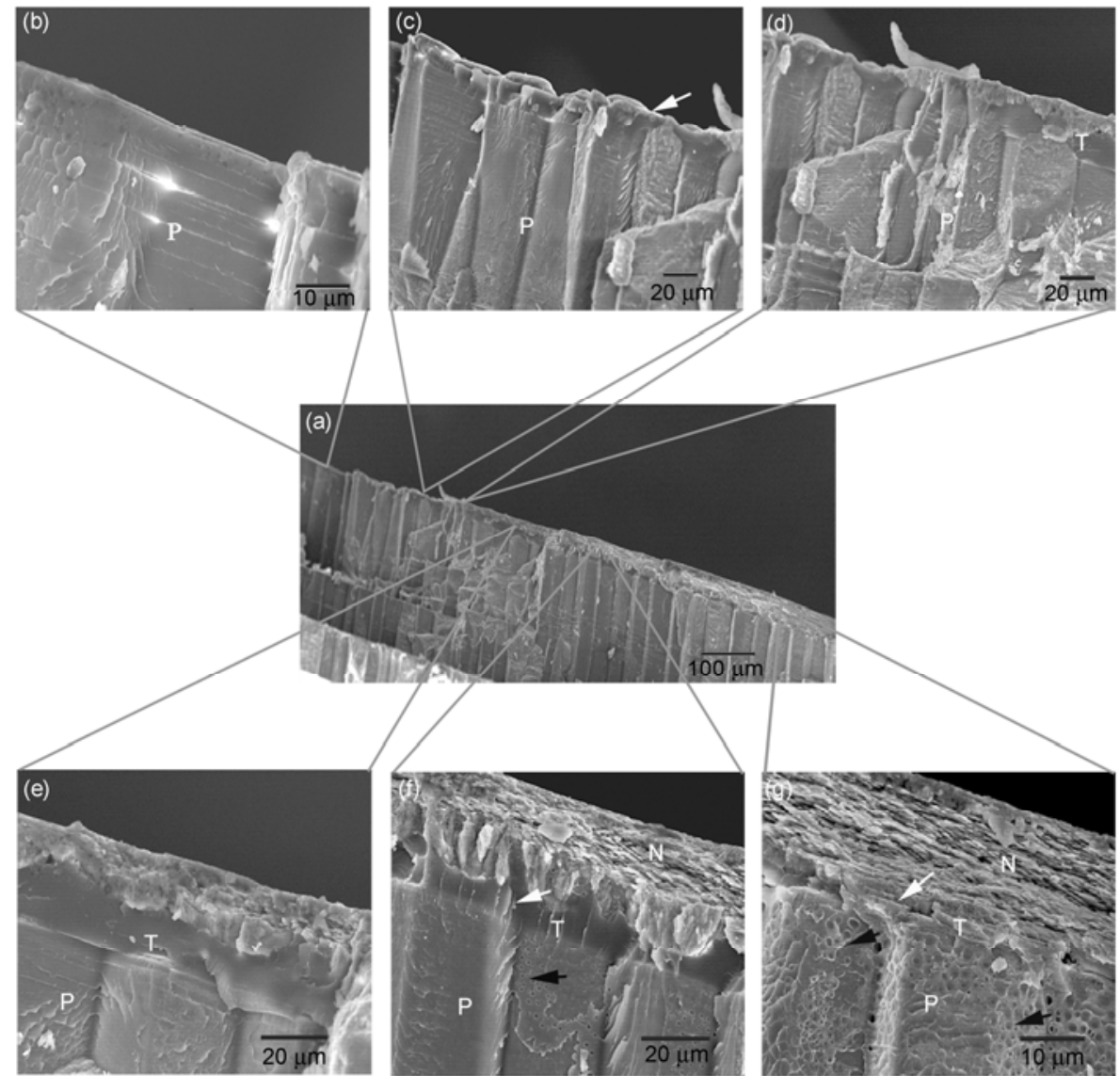

Figure 5 Cross-section of the shell showing the transition from the prismatic to the nacreous layer. (a) Cross-section of the shell at low magnification; (b) the prisms near the transition zone; (c) the addition of organic matrix to the surface of the prisms (white arrow) in the formation of the transition layer; (d) and (e) an organic layer is formed on the surface; (f) the nacreous layer is deposited; (g) a thick nacreous layer is formed. P, the calcitic prismatic layer; T, the nacre-prism transition layer; N, the aragonitic nacreous layer. White arrows in (f) and (g) show the reduced levels of organic matrix. The direction of growth of the shell is from right to left.

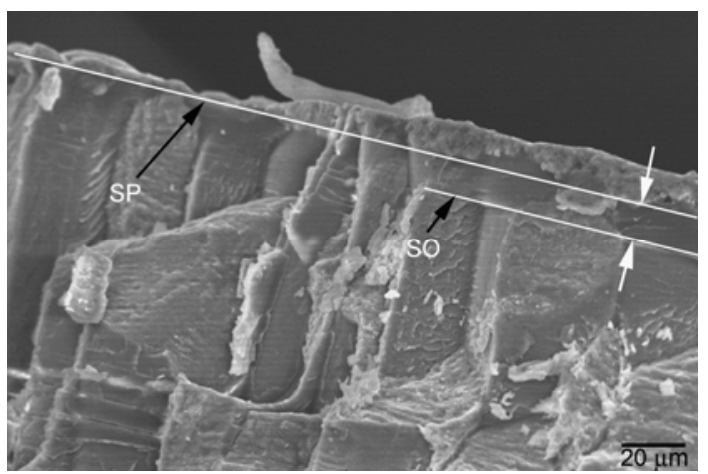

Figure 6 The organic layer formed under the surface of the nacre-prism transition layer. This figure is a labeled similarly to Figure 5(d). SP, the levels of the tops of the prisms in the prismatic layer; SO, the lower surface of the organic layer.

The calcitic prisms are arranged perpendicularly to the surface of the shell and are surrounded by interprismatic organic walls. Aragonite platelets of the nacreous layer are arranged in continuous parallel layers that are separated by interlamellar organic sheets and intercrystalline organic membranes [15-17]. The interaction between aragonite platelets and the organic matrix increases the fracture resistance of the nacreous layer [8]. The nacre-prism transition layer is essentially an organic sheet that joins the prismatic layer and nacreous layer. We propose that, although the crystal phase is discontinuous, the organic phase is continuous throughout the shell. Further, the continuum of the organic phase contributes to the strong adhesion between the prismatic and nacreous layers in the shell. To test this scenario, we examined the changes that occur in the organic matrix during the formation of the nacre-prism transition.

By examining 30 shells, we distinguished three different appearances of the nacre-prism transition layer related to the interprismatic organic envelopes. In the first type, the organic matrix secreted by the mantle was assembled in conjunction with the interprismatic organic envelopes (Figure 2(b) and (c)) rather than with the crystalline phase, implying continuous growth of the organic matrix. In the second type, the shapes of the prisms and their interprismatic organic envelopes changed within the transition zone, and the interprismatic organic envelopes became indistinguishable from 
the organic matrix. In the third type, the quantity of organic matrix in the interprismatic organic envelopes decreased near to the nacre-prism transition zone on the inner surface of the shell. However, the interprismatic organic envelopes seemed to be integrated into the organic matrix around the prisms at the point that the prisms disappeared in surface view (Figure 4(f)).

The variation in the appearance of the transition layer during its formation may reflect different growth rates of the shells. When shells grow slowly, the transition in the manner of growth is moderate, whereas when shells grow fast the transition becomes acute. In this study, we identified three types of nacre-prism transition formation, but more types might exist. However, we believe that the three types observed provide information that helps to elucidate the role of the organic matrix in the formation of the nacre-prism transition to some extent. During the formation of the nacre-prism transition layer, the organic matrix from this layer together with components of the interprismatic organic envelopes appears to be reassembled into a new structure. A key point is that although the changes occurring in the interprismatic organic envelopes were different, the organic phase was always continuous throughout the whole shell.

\subsection{Embedding of the organic matrix into the prismatic layer}

The organic matrix of the nacre-prism transition layer no doubt differs from that of the nacreous and prismatic layers but probably has components in common with both layers. Pif, a nacre-specific protein, had been shown to exist in the nacre-prism transition layer [18]. A prismatic layer-specific protein, Prisilikin-39, also exists in the transition layer [19]. A study by Dauphin et al. of the structure and composition of the nacre-prism transition layer also indicated that these layers are not sharply defined from each other [20]. Aragonite pellets were observed to form on the polycrystalline aragonites [21]. Therefore, a series of changes may take place in the composition of the organic matrix of the nacre-prism transition layer that induce the formation of the polycrystalline aragonites [14], rather than the formation of calcite or of aragonite pellets.

In the first type of transition layer, the calcite continued to grow and the diameter of the newly formed prisms became smaller (Figure 7(a)-(c)). This decrease was visible on the growth surface of the prisms and continued until the surface was covered by the organic matrix (Figure 2(a)). The newly secreted organic matrix inhibited calcite growth in the prismatic layer while the organic matrix of the interprismatic organic envelopes increased (Figure 7(b)). The continuous growth of prisms resulted in the invasion of organic matrix into the prismatic layer (Figure 7(c)). Previous studies have observed that because of the inhibition of the final stages of prism growth, the surface of the prisms under

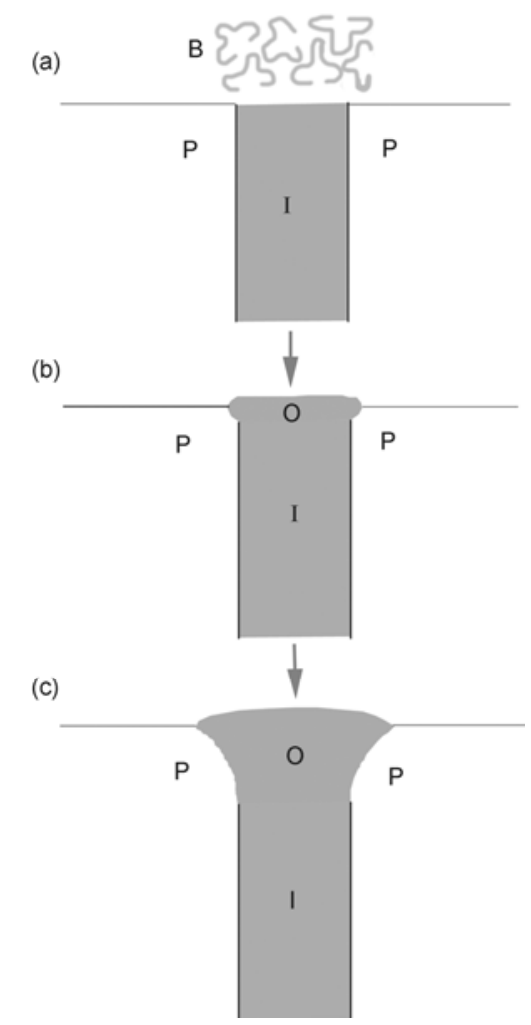

Figure 7 Inhibition of prism growth in the first type of nacre-prism transition layer formation. (a) Biomacromolecules are added to the surface of the interprismatic organic envelopes; (b) the increased organic matrix of the interprismatic organic envelopes begins to inhibit the growth of the prisms; (c) part of the organic matrix of the nacre-prism transition layer is embedded in the prismatic layer causing growth of the prismatic layer. B, biomacromolecules; $\mathrm{O}$, organic matrix; I, the interprismatic organic envelopes; $\mathrm{P}$, the calcite prismatic layer.

the nacreous layer is raised in cross sections of shells [20]. Inhibition of prism growth could explain the first type of appearance of nacre-prism transition layer but not the second and third types.

A reasonable explanation for the latter two scenarios is that the organic matrix from the nacre-prism transition layer penetrates into the organic phase below the surface of the prismatic layer. In the second type, the change in the shape of the prisms may be attributed to the rapid growth of the shell; the organic matrix embeds into the space that is created by the transformation of the prisms. Penetration of the prisms by the organic matrix could also explain the decreased quantity of organic matrix in interprismatic organic envelopes in the third type of transition layer formation. The apparent decrease in the organic matrix of the interprismatic organic envelopes may reflect a slower rate of addition of organic matrix to the surface of the interprismatic layer than in the first type (Figure 4(b)). However, the overgrowth of the prisms (Figure 4(c)) suggests that the crystalline component grew faster than the organic component before the formation of the nacre-prism transition layer. It should be noted that this area was very near the transition zone. If all 
of these changes occurred in the prismatic layer, it is unlikely that a nacre-prism transition layer composed solely of organic matrix could be formed in a short time. However, the thickness of the crystalline component decreased, and the organic part suddenly increased beside the overgrowth area (Figure 4(f)). After slight decalcification, the calcite overgrowths had fractured off from the prisms and were attached to the surface of the organic matrix surrounding the prisms (Figure 4(f), white arrows). Therefore, it appears that the rate of addition of the organic matrix to the surface of the interprismatic layer is rapid and that a series of changes must take place beneath the surface of the prismatic layer resulting in the formation of the nacre-prism transition layer. This hypothesis for the penetration of the organic matrix into the prismatic layer may help the understanding of other aspects of the process. As the organic matrix increases beneath the surface of prismatic layer, it may dissolve away part of the prisms, decreasing their diameter (Figure 8(a)(c)). However, on the surface of the prismatic layer, the overgrowing part of calcite covers part of the interprismatic

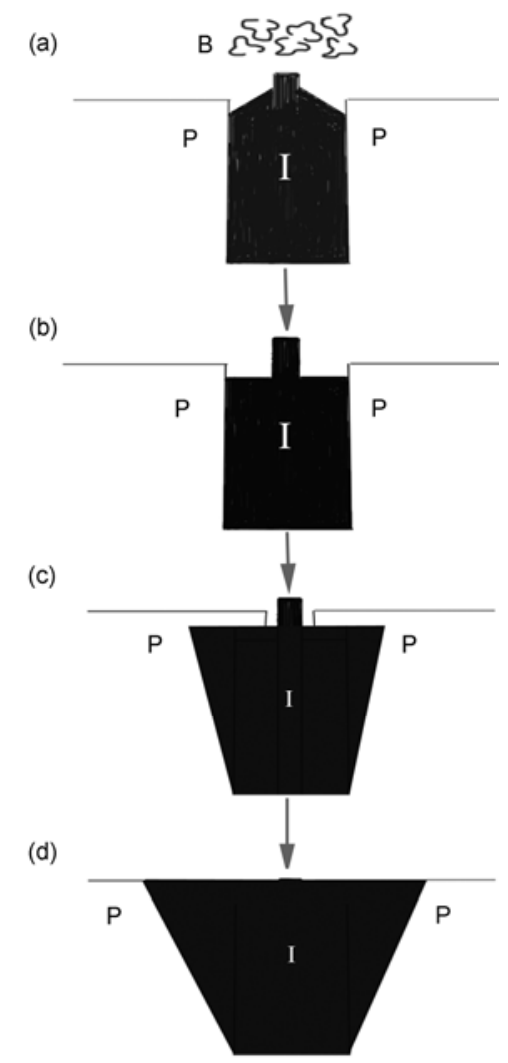

Figure 8 The change of the interprismatic organic envelopes in the third type of nacre-prism transition formation. (a) Biomacromolecules are added to the surface of the interprismatic organic envelopes; (b) the regions around the interprismatic organic envelopes become smooth and organic matrix flows on to them; (c) parts of the prisms are dissolved by the organic matrix and overgrowths from the prisms cover both sides of the interprismatic organic envelopes; (d) the overgrowths from the prisms are dissolved. B, biomacromolecules; I, the interprismatic organic envelopes; P, the calcite prismatic layer. organic envelopes (Figure 8(c)). Finally, the overgrowing calcite is dissolved (Figure 8(d)) and the visible diameter of prisms continues to decrease until the prismatic layer is covered by organic matrix.

In each variant of the formation of the nacre-prism transition layer, the organic matrix newly secreted by the mantle was embedded into the prismatic layer. The organic layer that is visible in cross-sections of the transition zone provides direct evidence for the presence of embedment (Figure 5(d) and (e)). The lower boundary of the organic layer is straight (Figure 5(d)-(f)) and is located below tops of the prisms near the nacre-prism transition zone (Figure 6). We propose that the organic lamella is not composed solely of organic matrix because the appearance of the organic layer is rapid and it is unlikely that the calcites could be eroded completely in such a short time. Instead, we suggest that it contains prisms embedded in macromolecules of the organic matrix newly secreted by the mantle. This organic material had a different morphology from the interprismatic organic matrix. The interaction between newly secreted organic matrix and the intrinsic matrix of the prismatic layer plays a key role, as they are reassembled into a new organic admixture. We propose that the embedment of this newly secreted organic matrix into the organic phase of the prismatic layer acts as a bond between the shell layers. This concept may stimulate new ideas for the design and synthesis of composite multilayered biomaterials.

The decreased thickness of the organic matrix in the nacre-prism transition layer was observed during nacreous layer formation and even after a thick nacreous layer covered the structure (Figure 5(e)- $(\mathrm{g})$ ). The reason for this phenomenon remains unknown. However, it is evident that this decrease results in the approach of the nacreous layer and the prismatic layer and this may assist in their adhesion.

\section{Conclusion}

During the formation of the nacre-prism transition layer in the shell of $P$. fucata, some of the newly secreted organic macromolecules cover the surface of the prisms to form the nacre-prism transition layer and some are embedded into the interprismatic organic envelopes to provide an adhesive. The prismatic and nacreous layers are closely bonded together by this interaction.

Special thanks are given to Mr. Liu Qiang and Mr. David Cushley for their help with the preparation of the manuscript. This work was supported by the National Natural Science Foundation of China (U0831001 and 40876068) and the National High Technology Research and Development Program of China (2010CB126405 and 2010AA- 09Z405).

1 Weiner S, Hood L. Soluble protein of the organic matrix of mollusk shells: A potential template for shell formation. Science, 1975, 190: 987-989 
2 Addadi L, Weiner S. Interactions between acidic proteins and crystals: Stereochemical requirements in biomineralization. Proc Natl Acad Sci USA, 1985, 82: 4110-4114

3 Belcher A M, Wu X H, Christensen R J, et al. Control of crystal phase switching and orientation by soluble mollusc-shell proteins. Nature, 1996, 381: 56-58

4 Falini G, Albeck S, Weiner S, et al. Control of aragonite or calcite polymorphism by mollusk shell macromolecules. Science, 1996, 271: 67-69

5 Levi Y, Albeck S, Brack A, et al. Control over aragonite crystal nucleation and growth: An in vitro study of biomineralization. Chem Eur J, 1998, 4: 389-396

6 Weiner S, Addadi L. Acidic macromolecules of mineralized tissues: The controls of crystal formation. Trends Biochem Sci, 1991, 16: 252256

7 Wheeler A P, George J W, Evans C A. Control of calcium carbonate nucleation and crystal growth by soluble matrix of oyster shell. Science, 1981, 212: 1397-1398

8 Smith B L, Schäffer T E, Viani M, et al. Molecular mechanistic origin of the toughness of natural adhesives, fibres and composites. Nature, 1999, 399: 761-763

9 Sudo S, Fujikawa T, Nagakura T, et al. Structures of mollusc shell framework proteins. Nature, 1997, 387: 563-564

10 Samata T, Hayashi N, Kono M, et al. A new matrix protein family related to the nacreous layer formation of Pinctada fucata. FEBS Lett, 1999, 462: 225-229

11 Miyashita T, Takagi R, Okushima M, et al. Complementary DNA cloning and characterization of pearlin, a new class of matrix protein in the nacreous layer of oyster pearls. Mar Biotechnol, 2000, 2: 409_ 418
12 Suzuki M, Murayama E, Inoue H, et al. Characterization of Prismalin-14, a novel matrix protein from the prismatic layer of the Japanese pearl oyster (Pinctada fucata). Biochem J, 2004, 382: 205-213

13 Tsukamoto D, Sarashina I, Endo K D. Structure and expression of an unusually acidic matrix protein of pearl oyster shells. Biochem Biophys Res Commun, 2004, 320: 1175-1180

14 Checa A G, Rodríguez-Navarro A B. Self-organisation of nacre in the shells of Pterioida (Bivalvia: Mollusca). Biomaterials, 2005, 26: 1071-1079

15 Grégoire C. Topography of the organic components in mother-ofpearl. J Biophys Biochem Cytol, 1957, 3: 797-808

16 Watabe N. Studies on shell formation, XI crystal-matrix relationships in the inner layers of mollusk shells. J Ultrastruct Res, 1965, 12: 351-370

17 Wise S W. Microarchitecture and mode of formation of nacre (mother-of-pearl) in pelecypods, gastropods and cephalopods. Eclogae Geol Helv, 1970, 63: 775-797

18 Suzuki M, Saruwatari K, Kogure T, et al. An acidic matrix protein, Pif, is a key macromolecule for nacre formation. Science, 2009, 325: 1388-1390

19 Kong Y, Jing G, Yan Z, et al. Cloning and characterization of Prisilkin-39, a novel matrix protein serving a dual role in the prismatic layer formation from the oyster Pinctada fucata. J Biol Chem, 2009, 284: 10841-10854

20 Dauphin Y, Ball A D, Cotte M, et al. Structure and composition of the nacre-prisms transition in the shell of Pinctada margaritifera (Mollusca, Bivalvia). Anal Bioanal Chem, 2008, 390: 1659-1669

21 Saruwatari K, Matsui T, Mukai H, et al. Nucleation and growth of aragonite crystals at the growth front of nacres in pearl oyster, Pinctada fucata. Biomaterials, 2009, 30: 3028-3034

Open Access This article is distributed under the terms of the Creative Commons Attribution License which permits any use, distribution, and reproduction in any medium, provided the original author(s) and source are credited. 